\title{
Gyroidal Niobium Sulfide/Carbon Hybrid Monoliths for Electrochemical Energy Storage
}

\author{
Simon Fleischmann, ${ }^{[a, b]}$ Tobias S. Dörr, ${ }^{[a, b]}$ Anna Frank, ${ }^{[c]}$ Stefan W. Hieke, ${ }^{[c]}$ David Doblas- \\ Jimenez, ${ }^{[a]}$ Christina Scheu, ${ }^{[c, d]}$ Peter W. de Oliveira, ${ }^{[a]}$ Tobias Kraus, ${ }^{[a, b]}$ and Volker Presser* ${ }^{[a, b]}$
}

\begin{abstract}
Transition metal dichalcogenides are attractive two-dimensional electrode materials for electrochemical energy storage devices due to their high reversible charge storage capacity. Hybridization of these materials with carbon promises enhanced performance by facilitating the access to reactive sites and extended mechanical stabilization. Herein, we introduce a $\mathrm{NbS}_{2} /$ $C$ hybrid material exhibiting a gyroidal microstructure synthesized through macromolecular co-assembly of a tailored block copolymer and an organometallic niobium precursor and subsequent sulfidation. Our synthesis allows the preparation of mechanically stable monoliths with $\mathrm{NbS}_{2}$ nanocrystals engulfed in a highly porous carbon shell. Due to the curvature of the gyroidal structure, abundant reactive sites are exposed that lead to an attractive performance in a lithium-containing electrolyte with a capacity of up to $400 \mathrm{mAh} / \mathrm{g}$.
\end{abstract}

Electrochemical energy storage devices are an integral building block of the transition toward sustainable energy sources. ${ }^{[1]}$ To date, lithium-ion batteries (LIBs) employing the rocking-chair insertion mechanism are the most technologically advanced devices and find widespread commercial application. ${ }^{[2]}$ However, the number of electron transfers per active material involved in insertion reactions is relatively small ( $1 \mathrm{Li}$ for $6 \mathrm{C}$, $<1$ per $\left.\mathrm{LiCoO}_{2}\right){ }^{[3]} \mathrm{A}$ shift to conversion or alloying reactions is essential to enable next-generation lithium batteries with increased energy densities. ${ }^{[4]}$ Promising electrode chemistries include metallic lithium, ${ }^{[5]}$ silicon, ${ }^{[6]}$ sulfur $^{[7]}$ or transition metal dichalcogenides (TMDs) ${ }^{[8]}$.

TMDs are two-dimensional materials with weak interlayer bonding that have attracted attention due to their optical and electronic properties. ${ }^{[9]}$ They have the structural formula $\mathrm{MX}_{2}$,

[a] Dr. S. Fleischmann, T. S. Dörr, Dr. D. Doblas-Jimenez, Dr. P. W. de Oliveira, Prof. Dr. T. Kraus, Prof. Dr. V. Presser

INM - Leibniz Institute for New Materials Campus D2 2, 66123 Saarbrücken, Germany E-mail: volker.presser@leibniz-inm.de

[b] Dr. S. Fleischmann, T. S. Dörr, Prof. Dr. T. Kraus, Prof. Dr. V. Presser Saarland University

Campus D2 2, 66123 Saarbrücken, Germany

[c] Dr. A. Frank, Dr. S. W. Hieke, Prof. Dr. C. Scheu Max-Planck-Institut für Eisenforschung $\mathrm{GmbH}$ Düsseldorf Nanoanalytics and Interfaces Max-Planck-Straße 1, 40237 Düsseldorf, Germany

[d] Prof. Dr. C. Scheu

Materials Analytics RWTH Aachen University Kopernikusstraße 10, 52074 Aachen, Germany

Supporting information for this article is available on the WWW under https://doi.org/10.1002/batt.201900035 where $\mathrm{M}$ is a transition metal (e.g., $\mathrm{Mo}, \mathrm{V}, \mathrm{Nb}, \mathrm{Ti}$ ) and $\mathrm{X}$ is a chalcogenide (i.e., $\mathrm{S}, \mathrm{Se}$, or Te). ${ }^{[9]}$ In particular, the electrochemical behavior of $\mathrm{MoS}_{2}$ as an anode in lithium- and sodiumion batteries was explored over the last few years. ${ }^{[8 b, 10]}$ It was found that the properties of TMD electrodes are highly dependent on the number and shape of the two-dimensional layers. ${ }^{[1]}$ Restacking of the two-dimensional layers is a common issue that leads to rapid performance degradation of TMD electrode materials. ${ }^{[12]}$ Direct hybridization of the electrochemically active material with a rationally structured carbon phase instead of the addition of conductive additive has been shown to improve the performance of electrode materials. ${ }^{[13]}$

Here, we introduce a three-dimensional, monolithic niobium disulfide/carbon hybrid material with a gyroidal microstructure synthesized via macromolecular co-assembly of a tailored block copolymer and an organometallic niobium precursor. Subsequent sulfidation in hydrogen and sulfurcontaining atmosphere yielded an ordered TMD with a simultaneously formed carbon shell. It is the first report on a gyroidal TMD/carbon hybrid material in literature. Few-layered $\mathrm{NbS}_{2}$ nanocrystals are engulfed in a microporous carbon shell to provide structural and mechanical integrity as well as electrical conductivity. The gyroidal structure with high surface curvature provides abundant active site exposure and good permeability for the electrolyte. ${ }^{[14]}$ It also stabilizes the structure to enable monolithic, three-dimensional electrodes, ${ }^{[15]}$ as was recently demonstrated for gyroidal $\mathrm{TiO}_{2} / \mathrm{C}$ monoliths. ${ }^{[16]}$ The attractiveness of such gyroidal electrode materials was further underlined by the recent demonstration of an ordered, threedimensional battery by the Wiesner group. ${ }^{[17]}$ The electrochemical properties of $\mathrm{NbS}_{2}$ were investigated by the Goodenough group, but only lithium insertion was observed, ${ }^{[18]}$ whereas lithium conversion reactions with increased capacities have only been demonstrated for transition metal- and selenium-doped $\mathrm{NbS}_{2}$ nanosheets. ${ }^{[19]}$ Our material shows, to the best of our knowledge, the first stable lithium conversion chemistry for monolithic $\mathrm{NbS}_{2}$ which we link to the high exposure of active sites from the gyroidal structure and the stability and electrical conductivity provided by the porous carbon shell. Most notably, macroscopic monolithic electrodes in cubic millimeter dimensions show attractive volumetric performances. As research on electrode materials with 3D hierarchical structures has recently gained significant attention, we believe that this novel class of materials holds the potential for combined high energy and power at a high material mass loading. ${ }^{[20]}$ 

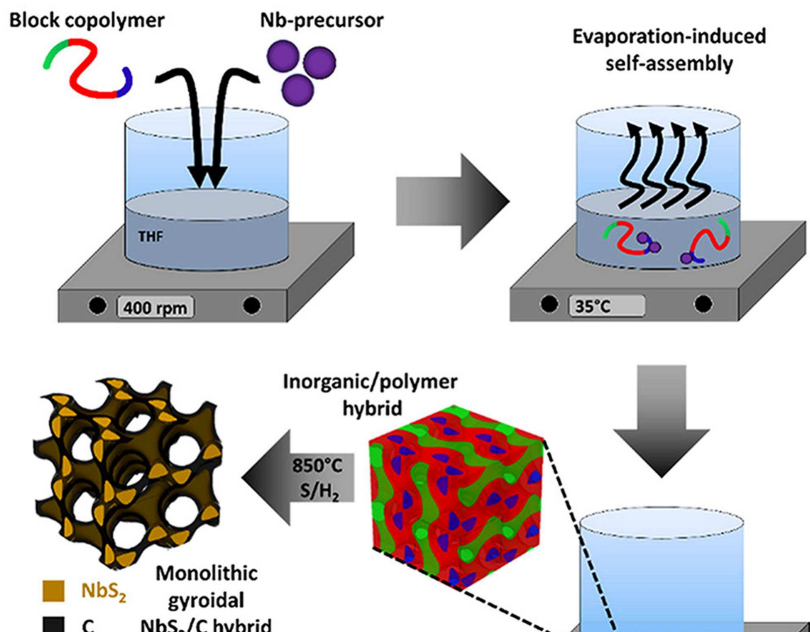

$\Rightarrow 10 \mathrm{~mm}$

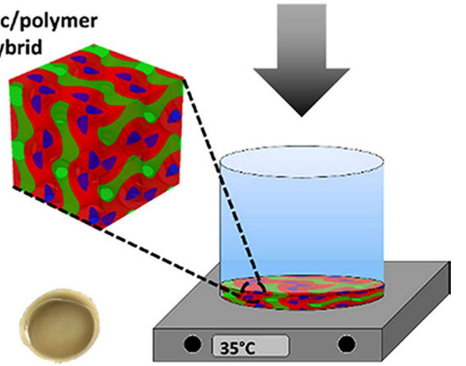

Figure 1. Synthesis scheme with photographs of both materials.

The synthesis of gyroidal niobium disulfide/carbon hybrid material was carried out by macromolecular co-assembly of poly(isoprene)-block-poly(styrene)-block-poly(ethylene oxide) (ISO) and niobium ethoxide as shown in Figure 1. The inorganic/polymer hybrid material was prepared by evaporation-induced self-assembly (EISA). It was further pyrolyzed and sulfidized by heat treatment under a hydrogen atmosphere with a sulfur source placed in the upstream side of the furnace to generate hydrogen sulfide gas. During the process, niobium disulfide is formed while simultaneously the organic polymer template is transformed into a carbon shell surrounding the niobium sulfide core. The carbon network stabilized the material and enabled a stable monolithic structure even after sulfidation that is accompanied by a substantial shrinking of the material.

The structure of the material was characterized by scanning electron microscopy (Figure 2A-B). The gyroidal structure is retained throughout the sample, forming mesoporous channels with a width of about $20-30 \mathrm{~nm}$. Scanning transmission electron micrographs with energy-dispersive X-ray (EDX) spectroscopy mappings were recorded to investigate the structure on the nanoscale and the elemental distribution inside the individual building blocks. The high angle annular dark field (HAADF) scanning electron microscopy (STEM) images and EDX mappings (Figure 2C-D) show that the ordered, gyroidal blocks consist of niobium and sulfur. Therefore, during sulfidation, niobium had remained in the gyroidal structure, and no restructuring upon sulfidation had occurred. The $\mathrm{NbS}_{2}$ nanocrystals are engulfed by a disordered carbon layer that originates from carbonization of the structure directing template, as can be seen in the annular bright field (ABF) STEM images (Figure 2E-F). Crucially, to form the curved gyroidal structure, the crystallites are frequently disrupted, thereby offering abundant access to reactive sites during electrochemical application.
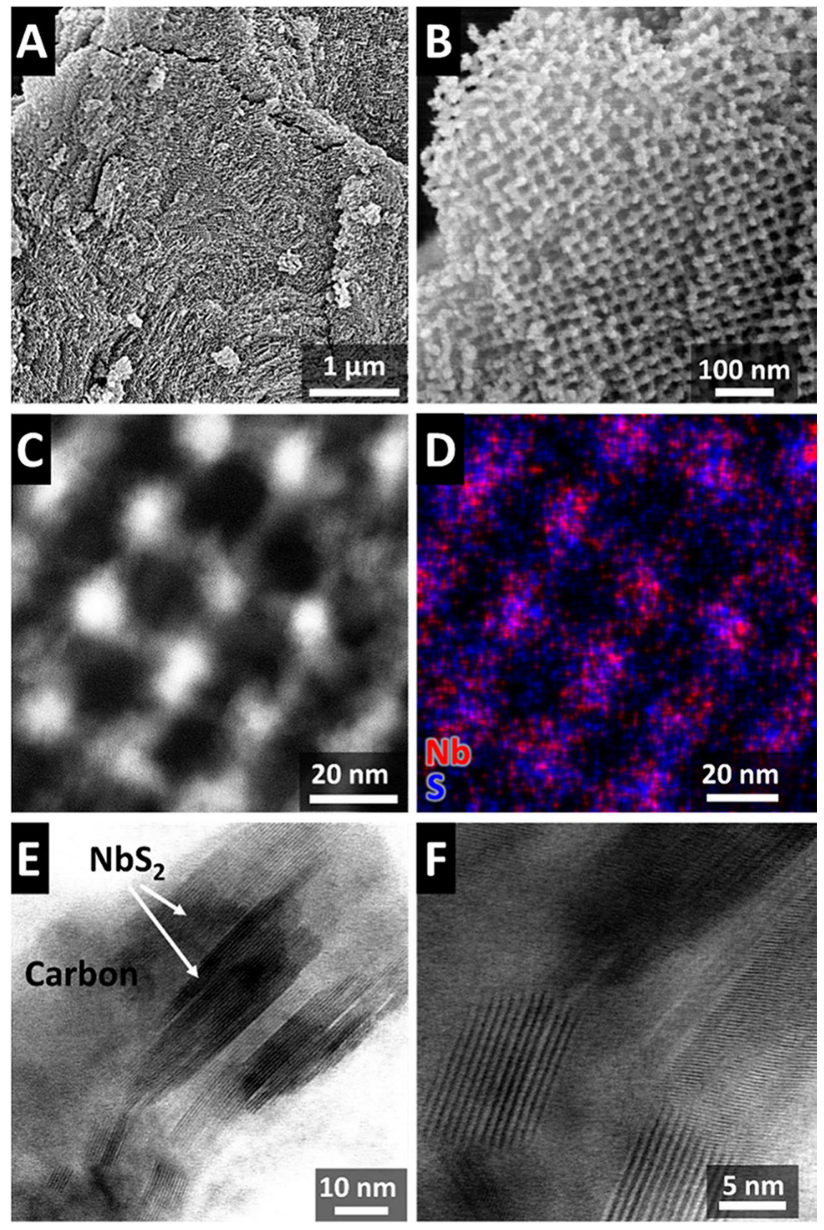

Figure 2. Scanning electron micrographs of gyro- $\mathrm{NbS}_{2} / \mathrm{C}$ hybrid material (A$B)$. HAADF STEM with corresponding EDX mapping (C-D), and ABF STEM images of one structure curvature region $(\mathrm{E}-\mathrm{F})$.

The X-ray diffractogram of the gyro- $\mathrm{NbS}_{2} / \mathrm{C}$ hybrid (Figure 3A) aligns with 3R-NbS 2 (PDF 38-1367; Supporting Information, Figure $\mathrm{S} 1)$. The broad reflections result from the nanocrystalline character of $\mathrm{NbS}_{2}$ with an approximate domain size of about $10 \mathrm{~nm}$ as determined by Rietveld refinement. 3R-NbS 2 is one of two polymorphs of niobium disulfide, next to $2 \mathrm{H}$ $\mathrm{NbS}_{2}$. Both form a hexagonal structure, with the unit cells of the $2 \mathrm{H}$ and $3 \mathrm{R}$ forms consisting of two or three $\mathrm{NbS}_{2}$ layers, respectively, that are weakly held together by van-der-Waals forces and offer vacant octahedral sites for lithium insertion. ${ }^{[18]}$ The absence of any graphite reflections in the diffractogram suggests that the carbon present in the gyro- $-\mathrm{NbS}_{2} / \mathrm{C}$ sample exhibits a disordered character.

We used Raman spectroscopy to further investigate the carbon phase in the hybrid material (Figure 3B). In the low Raman frequency range of $150-500 \mathrm{~cm}^{-1}$, typical signals of 3R$\mathrm{NbS}_{2}$ align with previous reports in the literature. ${ }^{[21]}$ Further, the typical D- and G-bands of carbon are found at $1333 \mathrm{~cm}^{-1}$ and $1600 \mathrm{~cm}^{-1}$, respectively. The mode positions and the calculated $\mathrm{I}_{D} / \mathrm{I}_{G}$ ratio (assuming Voigt peak shapes) of 0.55 confirming the disordered character and few-nm-domain size of carbon in the gyro- $\mathrm{NbS}_{2} / \mathrm{C}$ hybrid sample. ${ }^{[22]}$ 

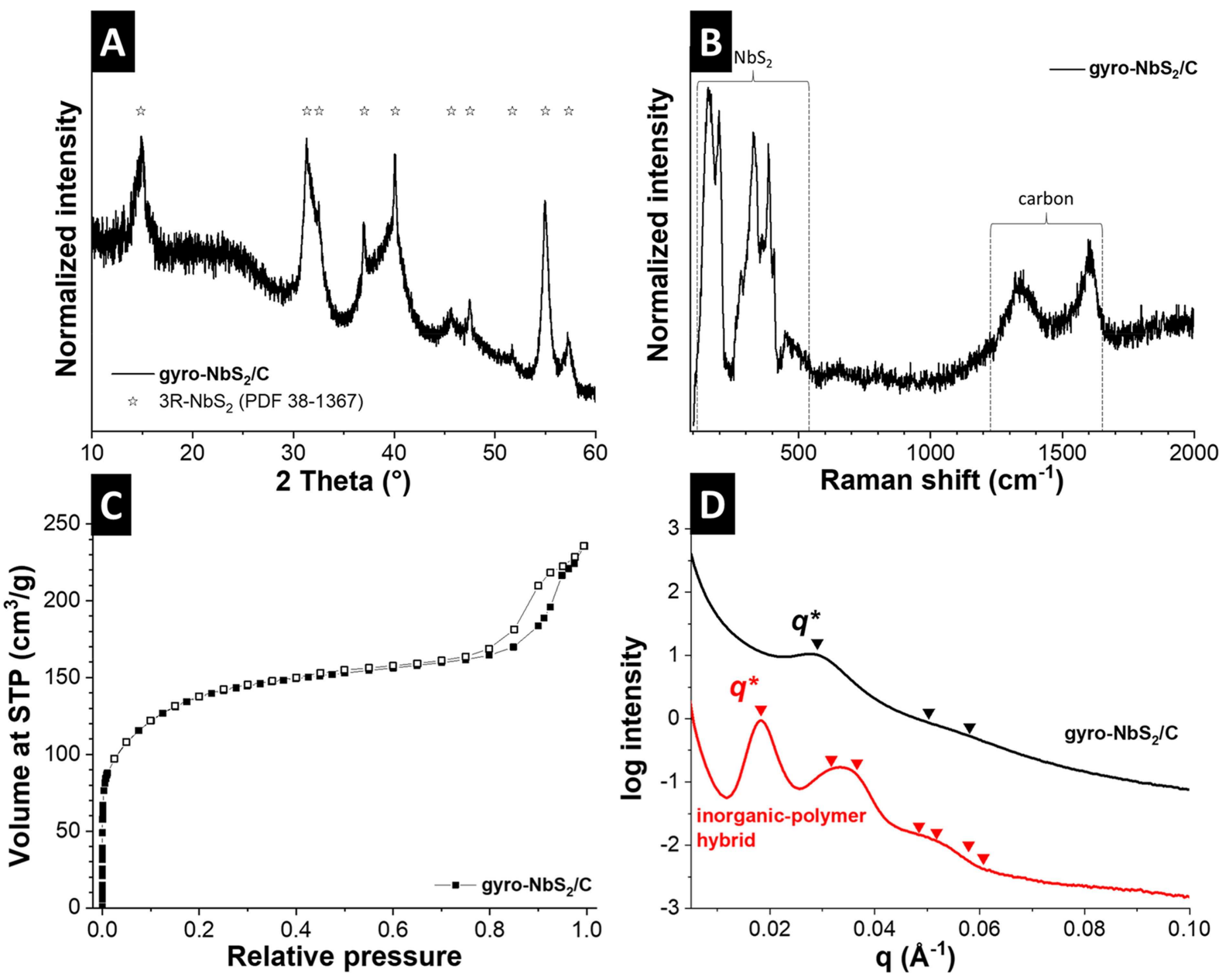

Figure 3. X-ray diffractogram (A), Raman spectrum (B), and nitrogen sorption isotherm (C) of the gyro- $\mathrm{NbS}_{2} / \mathrm{C}$ hybrid material. Small-angle $\mathrm{X}$-ray scattering data of inorganic-polymer hybrid and gyro- $-\mathrm{NbS}_{2} / \mathrm{C}$ hybrid material (D).

Thermogravimetric analysis was carried out to evaluate the amount of disordered carbon in the gyro- $\mathrm{NbS}_{2} / \mathrm{C}$ hybrid sample. It is shown that the composition of the gyro- $-\mathrm{NbS}_{2} / \mathrm{C}$ sample is 64 mass \% of $\mathrm{NbS}_{2}$ and 36 mass \% of disordered carbon (Supporting Information, Figure S2).

The porosity of the gyro- $-\mathrm{NbS}_{2} / \mathrm{C}$ hybrid sample was investigated by nitrogen gas sorption, and the nitrogen sorption isotherm is given in Figure 3C. The isotherm shape is a mixture of type I(b) and type IV(a) according to the classification of IUPAC. ${ }^{[23]}$ In the low-pressure region, there is a sudden increase in nitrogen uptake related to the filling of micropores of broad size distribution and narrow mesopores, resembling a type I(b) isotherm. ${ }^{[23]}$ This feature that is typical for activated carbons which are mainly comprised of micropores (pore size below $2 \mathrm{~nm}$ ) and small mesopores, strongly indicates a highly porous character of the disordered carbon shell engulfing the $\mathrm{NbS}_{2}$ nanocrystals. The specific surface area of the sample calculated by the two-dimensional non-local density functional theory (2D-NLDFT) ${ }^{[24]}$ is relatively high with around $390 \mathrm{~m}^{2} / \mathrm{g}$, further suggesting a high microporosity of the carbon shell. The pore volume of $0.33 \mathrm{~cm}^{3} / \mathrm{g}$ contains micro- and mesopores (Supporting Information, Figure S3) and is expected to be advantageous for the desired electrochemical application, as a high microporosity generally facilitates the access of lithium ions to the reactive sites of the $\mathrm{NbS}_{2}$ nanocrystals. In the highpressure regime, capillary condensation is observed with a clear hysteresis behavior that is related to larger mesopores forming bottle-necks, ${ }^{[23]}$ which originate from the mesoporous gyroidal structure of the sample.

The long-range order of the gyro- $\mathrm{NbS}_{2} / \mathrm{C}$ sample was further investigated by small-angle X-ray scattering (SAXS, Figure $3 D$ ). To identify the predominant microstructure, the positions of the first $\left(q^{*}\right)$ and subsequent higher order diffractions are compared to theoretically expected peak positions. From $q^{*}$, calculated positions for the alternating gyroid that can be identified for the samples are marked with triangles in Figure 3D. ${ }^{[25]}$ The experimental pattern of the inorganic/polymer hybrid agrees well with those predicted peak positions with $q^{*}$ that appears at $0.019 \AA^{-1}$, which corresponds to $33.1 \mathrm{~nm}$ in real space. The gyro- $-\mathrm{NbS}_{2} / \mathrm{C}$ also exhibits a characteristic scattering despite the harsh synthetic procedure, i.e., phase transition at high process temperatures for a free-standing, mesoporous inorganic material. This result is in good agreement with the data from the electron micro- 
scopy and gas sorption. The retained carbon shell is expected to mechanically stabilize the free-standing, mesoporous monolith and prevent extended pore condensation upon sulfidation. The shrinkage by about $37 \%$ of the microstructure during heat treatment aligns with a pronounced shift of $q^{*}$ to $0.03 \AA^{-1}$ $(20.9 \mathrm{~nm})$ for the gyro- $\mathrm{NbS}_{2} / \mathrm{C}$ compared to the hybrid sample.

We characterized gyro- $\mathrm{NbS}_{2} / \mathrm{C}$ hybrid electrodes in a halfcell setup versus metallic lithium. Galvanostatic charge/discharge curves are shown in Figure $4 \mathrm{~A}$ with cycling rates between $50 \mathrm{~mA} / \mathrm{g}$ and $5 \mathrm{~A} / \mathrm{g}$. The cathodic scan shows two characteristic regions: one in the range of $1.7-1.0 \mathrm{~V} \mathrm{vs} . \mathrm{Li} / \mathrm{Li}^{+}$, and one below $0.7 \mathrm{~V}$ vs. $\mathrm{Li} / \mathrm{Li}^{+}$. Previous works using $3 \mathrm{R}-\mathrm{NbS}_{2}$ in the potential region between $3.0 \mathrm{~V}$ and $1.0 \mathrm{~V}$ vs. $\mathrm{Li}^{\prime} \mathrm{Li}^{+}$reported on lithium insertion as the predominant charge storage mechanism with the formation of $\mathrm{Li}_{x} \mathrm{NbS}_{2}$, with $0<\mathrm{x}<1$. $^{[18]}$ The maximum insertion capacity of $1 \mathrm{Li}$ per $1 \mathrm{NbS}_{2}$ corresponds to a theoretical capacity of $170 \mathrm{mAh} / \mathrm{g} \cdot{ }^{[18]}$ This is in alignment with the gyro- $\mathrm{NbS}_{2} / \mathrm{C}$ hybrid electrode that exhibits a reversible capacity in the lithiation step of $107 \mathrm{mAh} / \mathrm{g}$ up to a potential of $1.0 \mathrm{~V} \mathrm{vs}$. $\mathrm{Li} / \mathrm{Li}^{+}$(corresponds to $167 \mathrm{mAh} / \mathrm{g}$ per NbS${ }_{2}$ ).

Further discharge to a potential of $0.05 \mathrm{~V}$ vs. $\mathrm{Li}^{2} \mathrm{Li}^{+}$at a rate of $50 \mathrm{~mA} / \mathrm{g}$ increases the specific capacity of the material to around $400 \mathrm{mAh} / \mathrm{g}$. The additional electron transfers imply that further redox reactions take place in this potential range. The literature suggests conversion reactions for materials with a similar structure like $3 \mathrm{R}-\mathrm{MoS}_{2}$ in this potential range. ${ }^{[8]}$ Recently, an investigation of the conversion reactions of codoped $\mathrm{Fe}_{0.3} \mathrm{Nb}_{0.7} \mathrm{~S}_{1.6} \mathrm{Se}_{0.4}$ suggested the irreversible conversion during the first cathodic scan of $\mathrm{NbS}_{2}$ to $\mathrm{NbS}$, and $\mathrm{NbS}$ to $\mathrm{Nb}$, while forming $\mathrm{Li}_{2} \mathrm{~S} .{ }^{[19]}$ The authors observed a sharp redox peak at $2.34 \mathrm{~V}$ vs. $\mathrm{Li}^{\prime} / \mathrm{Li}^{+}$during the first anodic scan that indicated the oxidation of $\mathrm{Li}_{2} \mathrm{~S}$ to $\mathrm{S}^{[26]}$ During the first cycle of the gyro$\mathrm{NbS}_{2} / \mathrm{C}$ material (Supporting Information, Figure $\mathrm{S} 4$ ), a small signal is detected at that potential during the anodic scan, suggesting only a small amount of the formed $\mathrm{Li}_{2} \mathrm{~S}$ reacted irreversibly. Anodic plateaus at $1.8 \mathrm{~V} \mathrm{vs.} \mathrm{Li} / \mathrm{Li}^{+}$of the gyro- $-\mathrm{NbS}_{2} /$ $\mathrm{C}$ material in the subsequent cycles are indicating reversible reactions between $\mathrm{Li}$ and $\mathrm{NbS}_{2}$.

Due to the large specific surface area of the gyro- $-\mathrm{NbS}_{2} / \mathrm{C}$ material, stable formation of the solid electrolyte interphase (SEI) in the potential range below $1 \mathrm{~V} \mathrm{vs.} \mathrm{Li}^{-\mathrm{Li}^{+}}$is of concern. ${ }^{[27]}$
Accordingly, we see a very low Coulombic efficiency of the system in the first cycle of $49.7 \%$. Yet, a stable SEI is readily formed, and we measured a Coulombic efficiency of $98.2 \%$ in the third cycle.

The rate handling behavior of the gyro- $\mathrm{NbS}_{2} / \mathrm{C}$ material is shown in Figure 4B. It can be seen that the material undergoes an irreversible capacity loss during the first few cycles. ${ }^{[19]}$ The material shows promising rate handling capability up to high currents of about $1 \mathrm{~A} / \mathrm{g}$, where capacity retention of around $160 \mathrm{mAh} / \mathrm{g}$ is observed. The Coulombic efficiency remains close to $100 \%$ for all cycling currents. The cycling stability of the material is tested at a rate of $100 \mathrm{~mA} / \mathrm{g}$, and after about 200 cycles (Figure $4 C$ ), capacity retention of about $50 \%$ of the initial value is observed, which can be linked to partially irreversible conversion reactions, as well as to the formation of a large SEI. ${ }^{[19]}$ Further, the restructuring of the material during conversion reactions can lead to a loss of contact with the conductive carbon phase, restricting further electron transfer to parts of the $\mathrm{NbS}_{2}$. Future work should include a systematic optimization of the carbon shell, which we expect to improve the cycling stability.

Finally, the free-standing, monolithic gyro- $\mathrm{NbS}_{2} / \mathrm{C}$ material was directly used as an electrode without the addition of any conductive additives or binder (Figure S5). The monolith electrodes provided maximum specific capacity of around $112 \mathrm{mAh} / \mathrm{g}$ at a rate of $50 \mathrm{~mA} / \mathrm{g}$. Considering a high average macroscopic bulk density of $1.4 \mathrm{~g} / \mathrm{cm}^{3}$, the thick monolith electrodes showed good volumetric performance beyond $150 \mathrm{mAh} / \mathrm{cm}^{3}$. The lower gravimetric capacity compared to the film-electrode can be explained by rather poor contacting the current collector due to the slightly curved monolith surface. We believe that this issue can be resolved by several strategies, such as sputter-coating of the current collector directly to the monolithic electrode or precision cutting of the monolith to achieve a flat surface. ${ }^{[28]}$

Monolithic $\mathrm{NbS}_{2} / \mathrm{C}$ hybrid materials with a mesoporous, gyroidal microstructure were synthesized by a macromolecular co-assembly of a tailored block copolymer and an organometallic niobium precursor and subsequent sulfidation. It is the first report of a TMD/C hybrid material with a gyroidal structure. $\mathrm{NbS}_{2}$ nanocrystals are engulfed by a conductive, porous carbon
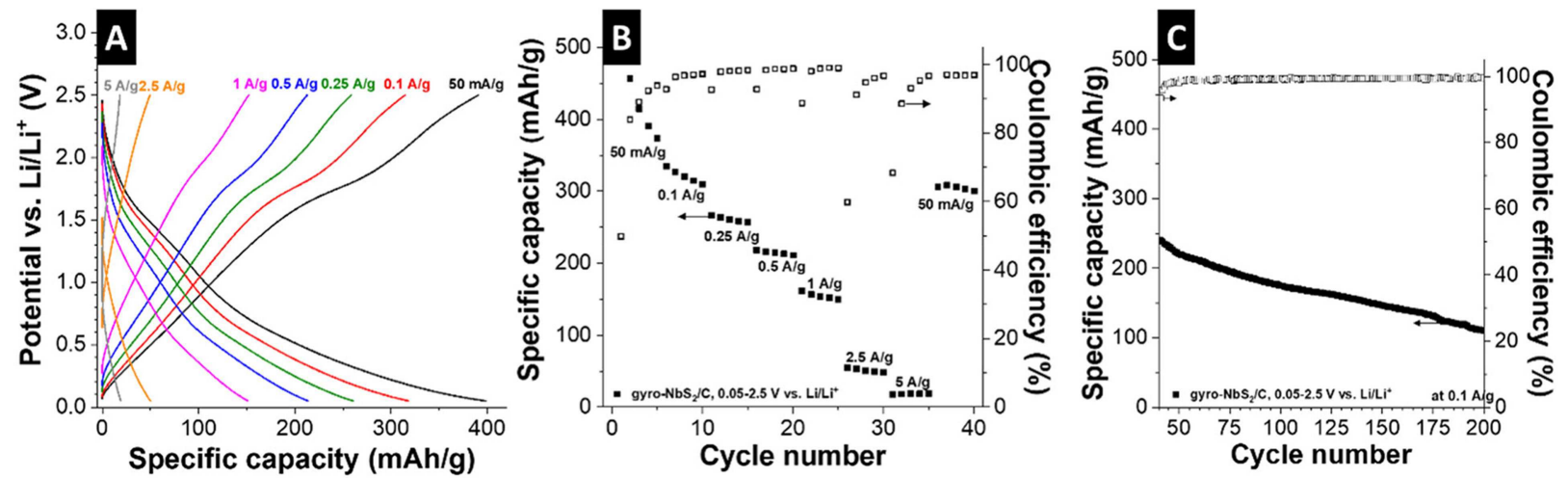

Figure 4. Galvanostatic charge/discharge potential profiles at currents between $50 \mathrm{~mA} / \mathrm{g}$ and $5 \mathrm{~A} / \mathrm{g}(\mathrm{A})$, galvanostatic rate handling data (B), and galvanostatic cycling stability test at $0.1 \mathrm{~A} / \mathrm{g}(\mathrm{C})$ of gyro- $\mathrm{NbS}_{2} / \mathrm{C}$ thin film electrodes. 
shell, also mechanically stabilizing the structure. The curvature of the gyroidal microstructure beneficially enables abundant access of lithium to reactive reaction sites. Casted film electrodes show reversible conversion reactions in lithium-containing half-cells with a maximum capacity of up to $400 \mathrm{mAh} / \mathrm{g}$. The direct use of the monolithic material as an electrode provided a high volumetric capacity up to $150 \mathrm{mAh} / \mathrm{cm}^{3}$. Our initial proofof-concept study on gyroidal TMD materials for electrochemical energy storage devices highlights the advantages of this microstructure and provides an attractive avenue for further research. Improvements related to the carbon shell are expected to improve the cycling stability further, and other TMD materials can be prepared by adjusting the synthesis protocol.

\section{Acknowledgements}

The work was part of the Carbon Metal Oxide Nanohybrid project (CarMON) supported by the Leibniz Association (SAW-2017). We thank Eduard Arzt (INM) for his continuing support. We thank Benjamin Krüner for gas sorption measurements, and Aura Tolosa and Pattarachai Srimuk (all INM) for helpful scientific discussions.

\section{Conflict of Interest}

The authors declare no conflict of interest.

Keywords: conversion reaction - electrochemical energy storage - lithium-ion batteries - materials science $\cdot$ transition metal dichalcogenide

[1] Z. Yang, J. Zhang, M. C. W. Kintner-Meyer, X. Lu, D. Choi, J. P. Lemmon, J. Liu, Chem. Rev. 2011, 111, 3577-3613.

[2] a) M. Armand, J. M. Tarascon, 2008, 451, 652-657; b) J. B. Goodenough, K. S. Park, J. Am. Chem. Soc. 2013, 135, 1167-1176.

[3] N.-S. Choi, Z. Chen, S. A. Freunberger, X. Ji, Y.-K. Sun, K. Amine, G. Yushin, L. F. Nazar, J. Cho, P. G. Bruce, Angew. Chem. Int. Ed. 2012, 51, 9994-10024; Angew. Chem. 2012, 124, 10134-10166.
[4] aY. Liu, G. Zhou, K. Liu, Y. Cui, Acc. Chem. Res. 2017, 50, 2895-2905; bN. Nitta, F. Wu, J. T. Lee, G. Yushin, Mater. Today 2015, 18, 252-264.

[5] D. Lin, Y. Liu, Y. Cui, Nat. Nanotechnol. 2017, 12, 194.

[6] X. Su, Q. Wu, J. Li, X. Xiao, A. Lott, W. Lu, B. W. Sheldon, J. Wu, Adv. Energy Mater. 2014, 4, 1300882.

[7] X. Ji, L. F. Nazar, J. Mater. Chem. 2010, 20, 9821-9826.

[8] a) M. S. Whittingham, Prog. Solid State Chem. 1978, 12, 41-99; b) T. Stephenson, Z. Li, B. Olsen, D. Mitlin, Energy Environ. Sci. 2014, 7, 209231.

[9] Q. H. Wang, K. Kalantar-Zadeh, A. Kis, J. N. Coleman, M. S. Strano, Nat Nanotechnol. 2012, 7, 699.

[10] G. Wang, J. Zhang, S. Yang, F. Wang, X. Zhuang, K. Müllen, X. Feng, Adv. Energy Mater. 2018, 8, 1702254.

[11] G. A. Muller, J. B. Cook, H.-S. Kim, S. H. Tolbert, B. Dunn, Nano Lett. 2015, 15, 1911-1917.

[12] Z. Deng, H. Jiang, C. Li, Small 2018, 14, 1800148.

[13] S. Fleischmann, A. Tolosa, V. Presser, Chem. Eur. J. 2018, 24, 1214312153.

[14] J. Kibsgaard, Z. Chen, B. N. Reinecke, T. F. Jaramillo, Nat. Mater. 2012, $11,963$.

[15] Y. Gogotsi, P. Simon, Science 2011, 334, 917-918.

[16] T. S. Dörr, S. Fleischmann, M. Zeiger, I. Grobelsek, P. W. de Oliveira, V. Presser, Chem. Eur. J. 2018, 24, 6358-6363.

[17] J. G. Werner, G. G. Rodríguez-Calero, H. D. Abruña, U. Wiesner, Energy Environ. Sci. 2018, 11, 1261-1270.

[18] Y. Liao, K.-S. Park, P. Singh, W. Li, J. B. Goodenough, J. Power Sources 2014, 245, 27-32.

[19] J. Zhang, C. Du, Z. Dai, W. Chen, Y. Zheng, B. Li, Y. Zong, X. Wang, J. Zhu, Q. Yan, ACS Nano 2017, 11, 10599-10607.

[20] H. Sun, J. Zhu, D. Baumann, L. Peng, Y. Xu, I. Shakir, Y. Huang, X. Duan, Nat. Rev. Mater. 2018, 4, 45-60.

[21] C. J. Carmalt, E. S. Peters, I. P. Parkin, T. D. Manning, A. L. Hector, Eur. J. Inorg. Chem. 2004, 2004, 4470-4476.

[22] G. A. Zickler, B. Smarsly, N. Gierlinger, H. Peterlik, O. Paris, Carbon 2006, 44, 3239-3246.

[23] M. Thommes, K. Kaneko, A. V. Neimark, J.P. Olivier, F. RodriguezReinoso, J. Rouquerol, K. S. W. Sing, Pure Appl. Chem. 2015, 87, 1051 1069.

[24] J. Jagiello, J. P. Olivier, Carbon 2013, 55, 70-80.

[25] T. H. Epps, E. W. Cochran, C. M. Hardy, T. S. Bailey, R. S. Waletzko, F. S. Bates, Macromolecules 2004, 37, 7085-7088.

[26] Z. Zhang, H. Zhao, Y. Teng, X. Chang, Q. Xia, Z. Li, J. Fang, Z. Du, K. Swierczek, Adv. Energy Mater. 2018, 8, 1700174.

[27] P. Verma, P. Maire, P. Novák, Electrochim. Acta 2010, 55, 6332-6341.

[28] J. Busom, A. Schreiber, A. Tolosa, N. Jäckel, I. Grobelsek, N. J. Peter, V. Presser, J. Power Sources 2016, 329, 432-440.

Manuscript received: February 28, 2019

Revised manuscript received: April 5, 2019

Accepted manuscript online: April 8, 2019

Version of record online: April 23, 2019 